\title{
QUASIEQUATIONAL THEORIES OF FLAT ALGEBRAS
}

\author{
J. JeŽEK, Praha, M. Maróti and R. MCKenzie, Nashville
}

(Received October 16, 2002)

\begin{abstract}
We prove that finite flat digraph algebras and, more generally, finite compatible flat algebras satisfying a certain condition are finitely $q$-based (possess a finite basis for their quasiequations). We also exhibit an example of a twelve-element compatible flat algebra that is not finitely $q$-based.
\end{abstract}

Keywords: quasiequation, flat algebra

MSC 2000: 08C15, 08B05

\section{INTRODUCTION}

For a finite directed graph $(V, E)$ one can define an algebra with the underlying set $V \cup E \cup\{0\}$, one constant 0 and two binary operations $\wedge$, - in this way: $a \wedge a=a$ and $a \wedge b=0$ whenever $a \neq b ; a b=c$ whenever $a, c \in V$ and $b=(a, c) \in E$; $a b=0$ in all other cases. Algebras obtained from finite directed graphs in this way are called finite flat digraph algebras. One particular six-element flat digraph algebra (inherently non-finitely based for equations) played a significant role in the proof of undecidability of the existence of a finite basis for the equational theory of a finite algebra ([2], [3] and [4]). It was plausible to expect that it could serve a similar purpose in an attempt to prove that also the existence of a finite basis for the quasiequations of a finite algebra is undecidable. However, in this paper we are going to show that all finite flat digraph algebras are finitely $q$-based (possess a finite basis for their uasiequations), which makes them unsuitable. We will investigate a more

The first author was partially supported by the grant \# 201/02/0594 of the Grant Agency of the Czech Republic, and by the Institutional grant MSM0021620839; the second author was partially supported by the grant No. T $\backslash 37877$ of the Hungarian National Foundation for Scientific Research (OTHA); the third author was supported by the NSF grant \# DMS-9971352. 
general class of finite compatible flat algebras, in which (under a modest assumption on the signature) every algebra can be embedded both into a finitely $q$-based and into a non-finitely $q$-based algebra.

For the terminology and basic concepts of universal algebra the reader is referred to the monograph [5]. For the literature on quasiequational theories see, e.g., [1] and [6].

\section{Compatible 0-Semilattice algebras}

Let $\sigma$ be a finite signature containing (among other symbols) a binary symbol $\wedge$ (the meet) and a nullary symbol 0 .

By a 0 -semilattice algebra we mean a $\sigma$-algebra satisfying the equations

(1) $x \wedge(y \wedge z)=(x \wedge y) \wedge z$,

(2) $x \wedge y=y \wedge x$,

(3) $x \wedge x=x$,

(4) $f\left(x_{1}, \ldots, x_{i-1}, 0, x_{i+1}, \ldots, x_{n}\right)=0$ for every $n$-ary operation $f$ of $\sigma$ and every $i \in\{1, \ldots, n\}$.

A 0 -semilattice algebra is said to be compatible if it satisfies the equations

(5) $f\left(z_{1}, \ldots, z_{i-1}, x \wedge y, z_{i+1}, \ldots, z_{n}\right)=f\left(z_{1}, \ldots, z_{i-1}, x, z_{i+1}, \ldots, z_{n}\right) \wedge f\left(z_{1}, \ldots\right.$, $\left.z_{i-1}, y, z_{i+1}, \ldots, z_{n}\right)$ for every $n$-ary operation $f$ of $\sigma$ and every $i \in\{1, \ldots, n\}$.

So, the class of compatible 0 -semilattice $\sigma$-algebras is a variety.

For a variable $x$, basic $x$-terms of depth $n$ are defined as follows. The term $x$ is the only basic $x$-term of depth 0 . For $n>0$, basic $x$-terms of depth $n$ are the terms $f\left(x_{1}, \ldots, x_{i-1}, t, x_{i+1}, \ldots, x_{n}\right)$ such that $f$ is an $n$-ary operation of $\sigma, 1 \leqslant i \leqslant n$, $t$ is a basic $x$-term of depth $n-1$ and $x_{1}, \ldots$ are variables different from $x$. A basic $x$-term $t$ will be usually denoted by $t(x)$, in which case $t(u)$ stands for the term resulting from $t$ by substituting $u$ for $x$ (where $u$ is any term).

For a $\sigma$-algebra $B$ and a basic $x$-term $t$ of depth $n$, any interpretation of the variables different from $x$ by elements of $B$ gives rise to a unary polynomial of $B$. The unary polynomials obtained in this way will be called the basic polynomials of $B$ of depth $n$.

Lemma 2.1. Let $A$ be a compatible 0-semilattice algebra. Then $p(a \wedge b)=$ $p(a) \wedge p(b)$ for all basic polynomials $p$ of $A$ and all elements $a, b \in A$.

Proof. It is easy. (Observe that the statement is not true for all unary polynomials $p$.) 
Lemma 2.2. Let $A$ be a compatible 0-semilattice algebra and $F$ be a proper filter of $A$ (i.e., a nonempty subset closed under meet, not containing 0 and such that $b \in F$ whenever $a \in F$ and $a \leqslant b$ ). Then for every basic polynomial $p$ of $A$, $p^{-1}(F)$ is either empty or a proper filter of $A$.

P r o of. It follows easily from Lemma 2.1.

By a flat algebra we mean a 0 -semilattice algebra $A$ such that $a \wedge b=0$ for all pairs of distinct elements $a, b \in A$. Observe that a flat algebra is monotonic, i.e., satisfies $x \leqslant y \rightarrow f\left(z_{1}, \ldots, z_{i-1}, x, z_{i+1}, \ldots, z_{n}\right) \leqslant f\left(z_{1}, \ldots, z_{i-1}, y, z_{i+1}, \ldots, z_{n}\right)$ for every $n$-ary operation $f$ of $\sigma$ and every $i \in\{1, \ldots, n\}$.

One can easily see that a flat algebra is compatible if and only if $\left(5^{\prime}\right) f\left(c_{1}, \ldots, c_{i-1}, a, c_{i+1}, \ldots, c_{n}\right)=f\left(c_{1}, \ldots, c_{i-1}, b, c_{i+1}, \ldots, c_{n}\right) \neq 0$ implies $a=b$ for every $n$-ary operation $f$ of $\sigma$ and every $i \in\{1, \ldots, n\}$.

For every partial algebra $G$ of a signature $\tau$ not containing $\wedge$ and 0 we can define a flat $\tau \cup\{\wedge, 0\}$-algebra $A$, called the flat algebra over $G$, by $A=G \cup\{0\}$, $f\left(a_{1}, \ldots, a_{n}\right)=a$ in $A$ whenever $f\left(a_{1}, \ldots, a_{n}\right)=a$ in $G$, and $f\left(a_{1}, \ldots, a_{n}\right)=0$ otherwise. This flat algebra is not necessarily compatible. For example, if $G$ is a finite groupoid, then the flat algebra over $G$ is compatible if and only if $G$ is a quasigroup. Finite flat digraph algebras are all compatible.

Observation 2.3. For every finite compatible flat algebra $A$ there exists a firstorder sentence $\Phi$ such that the finite models of $\Phi$ are precisely the finite algebras belonging to the quasivariety generated by $A$.

Proof. Put $K=|A|$. It is easy to see that the following are equivalent for a finite compatible 0 -semilattice algebra $B$ :

(e1) $B$ belongs to the quasivariety generated by $A$;

(e2) every two elements $b_{0}, b_{1}$ of $B$ such that $b_{0}<b_{1}$ can be separated by a congruence of $B$, the factor by which is isomorphic to a subalgebra of $A$;

(e3) for every $b_{0}, b_{1} \in B$ with $b_{0}<b_{1}$ there exist elements $c_{1}, \ldots, c_{r} \in B$ for some $r<K$ such that the principal filters $F_{1}, \ldots, F_{r}$ generated by $c_{1}, \ldots, c_{r}$ are pairwise disjoint, $b_{1} \in F_{1}, b_{0}$ belongs to the complement $O$ of $F_{1} \cup \ldots \cup F_{r}$ in $B$, the equivalence $R$ with blocks $O, F_{1}, \ldots, F_{r}$ is a congruence of $B$ and the factor $B / R$ is isomorphic to a subalgebra of $A$.

Clearly, the condition (e3) can be rewritten as a first-order sentence. 


\section{The QUASIVARIETY $Q_{A}^{\prime}$}

In the following let $A$ be a finite compatible, flat algebra. Put $K=|A|$.

Denote by $Q_{A}^{\prime}$ the quasivariety determined by the equations (1)-(5) and the following quasiequations:

(6) $x_{0} \leqslant x_{1} \& t(x) \geqslant x_{1} \& u(x) \geqslant x_{1} \& t(y) \geqslant x_{1} \& u(y) \wedge x_{1} \leqslant x_{0} \rightarrow x_{0}=x_{1}$ for every pair of basic $x$-terms $t, u$ of depth $\leqslant K$;

(7) $x_{0} \leqslant x_{1} \& H_{t_{1}, \ldots, t_{K}} \rightarrow x_{0}=x_{1}$ for every $K$-tuple of basic $x$-terms $t_{1}, \ldots, t_{K}$ of depth $\leqslant K$, where $H_{t_{1}, \ldots, t_{K}}$ is the conjunction of the following equations:

$$
\begin{aligned}
t_{i}\left(x_{i}\right) \geqslant x_{1} \quad(i=1, \ldots, K), \\
t_{i}\left(x_{j}\right) \wedge x_{1} \leqslant x_{0} \quad(i, j=1, \ldots, K \quad \text { and } i \neq j) .
\end{aligned}
$$

Lemma 3.1. $Q_{A}^{\prime}$ is a finitely $q$-based quasivariety containing $A$.

Proof. The set of quasiequations (6)-(7) is essentially finite, as it contains only finitely many quasiequations that differ by not only renaming their variables. Consequently, $Q_{A}^{\prime}$ is finitely $q$-based. It remains to prove that (6) and (7) are satisfied in $A$. Suppose that (6) fails in $A$ by some interpretation $v \mapsto v^{\prime}$ of variables. Then $x_{0}^{\prime}<x_{1}^{\prime}$, so that $x_{0}^{\prime}=0$; now $t\left(x^{\prime}\right) \geqslant x_{1}^{\prime}$ implies $t\left(x^{\prime}\right)=x_{1}^{\prime}$. Similarly we get $u\left(x^{\prime}\right)=x_{1}^{\prime}$ and $t\left(y^{\prime}\right)=x_{1}^{\prime}$. But $A$ satisfies $\left(5^{\prime}\right)$, so $t\left(x^{\prime}\right)=t\left(y^{\prime}\right) \neq 0$ implies $x^{\prime}=y^{\prime}$; hence $x_{1}^{\prime}=u\left(x^{\prime}\right) \wedge x_{1}^{\prime}=u\left(y^{\prime}\right) \wedge x_{1}^{\prime}=0$, a contradiction. Using the fact that $A$ cannot contain $K$ nonzero, pairwise distinct elements, one can similarly prove that $A$ satisfies the quasiequations (7).

Lemma 3.2. Let $B \in Q^{\prime}(A)$ and $b_{0}, b_{1} \in B$ be two elements such that $b_{1} \nless b_{0}$; let $F$ be a maximal filter of $B$ such that $b_{1} \in F$ and $b_{0} \notin F$. For any two basic polynomials $p, q$ of $B$ of depth $\leqslant K$, the sets $p^{-1}(F)$ and $q^{-1}(F)$ are either disjoint or equal.

Proof. The two basic polynomials $p$ and $q$ correspond to two basic terms $t$ and $u$ of depth $\leqslant K$. Suppose that there exist elements $x^{\prime}, y^{\prime}$ such that $p\left(x^{\prime}\right) \in F$, $p\left(y^{\prime}\right) \in F, q\left(x^{\prime}\right) \in F$ and $q\left(y^{\prime}\right) \notin F$. It follows from the maximality of $F$ that there exists an element $e \in F$ with $q\left(y^{\prime}\right) \wedge e \leqslant b_{0}$. Put $x_{1}^{\prime}=p\left(x^{\prime}\right) \wedge p\left(y^{\prime}\right) \wedge q\left(x^{\prime}\right) \wedge e$, so that $x_{1}^{\prime} \in F$. Put $x_{0}^{\prime}=b_{0} \wedge x_{1}^{\prime}$, so that $x_{0}^{\prime}<x_{1}^{\prime}$. But the quasiequation (e6) interpreted by $x \mapsto x^{\prime}, y \mapsto y^{\prime}, x_{0} \mapsto x_{0}^{\prime}, x_{1} \mapsto x_{1}^{\prime}$ gives $x_{0}^{\prime}=x_{1}^{\prime}$, a contradiction. 
Lemma 3.3. Let $B \in Q^{\prime}(A)$ and $b_{0}, b_{1} \in B$ be two elements such that $b_{1} \nless b_{0}$; let $F$ be a maximal filter of $B$ such that $b_{1} \in F$ and $b_{0} \notin F$. There are at most $K-1$ nonempty subsets of $B$ that can be expressed as $q^{-1}(F)$ for a basic polynomial $q$ of $B$, and they can be arranged into a sequence $F_{1}, \ldots, F_{r}$ (for some $r<K$ ) in such a way that $F_{1}=F$ and for every $i \in\{2, \ldots, r\}$ there are an index $j \in\{1, \ldots, i-1\}$ and a basic polynomial $p_{i}$ of $B$ of depth 1 with $F_{i}=p_{i}^{-1}\left(F_{j}\right)$. The collection $F_{1}, \ldots, F_{r}$, together with the complement of their union, is a partition and the corresponding equivalence is a congruence of $B$.

P r o of. Let us define a (finite or infinite) sequence $F_{1}, p_{1}, F_{2}, p_{2}, \ldots$ of filters $F_{i}$ and basic polynomials $p_{i}$ of depth $\leqslant 1$ by induction in this way: $F_{1}=F$ and $p_{1}$ is the identity on $B$; if $F_{i}, p_{i}$ have been defined and if there exist an element $a \notin F_{1} \cup \ldots \cup F_{i}$ and a basic polynomial $p$ of depth 1 such that $p(a) \in F_{j}$ for some $j \leqslant i$, take one such pair $a, p$ and put $p_{i+1}=p$ and $F_{i+1}=p_{i+1}^{-1}\left(F_{j}\right)$; if there is no such pair $a, p$, the sequence already constructed will have no continuation. Clearly (by induction on $i$ ), $F_{i}=q_{i}^{-1}(F)$ for a basic polynomial $q_{i}$ of $B$ of depth $<i$. The sets $F_{i}$ are pairwise disjoint filters according to Lemmas 2.2 and 3.2.

Suppose that the sequence has at least $K$ members $F_{1}, \ldots, F_{K}$. For any $i=$ $1, \ldots, K$ take an element $x_{i}^{\prime} \in F_{i}$, so that $q_{i}\left(x_{i}^{\prime}\right) \in F$. For every $i \neq j$ we have $x_{j}^{\prime} \notin F_{i}$, i.e., $q_{i}\left(x_{j}^{\prime}\right) \notin F$, so that there exists an element $e_{i, j} \in F$ with $q_{i}\left(x_{j}^{\prime}\right) \wedge e_{i, j} \leqslant b_{0}$. There is an element $x_{1}^{\prime} \in F$ such that $x_{1}^{\prime} \leqslant q_{i}\left(x_{i}^{\prime}\right)$ for all $i$ and $x_{1}^{\prime} \leqslant e_{i, j}$ for all $i \neq j$. Put $x_{0}^{\prime}=b_{0} \wedge x_{1}^{\prime}$, so that $x_{0}^{\prime}<x_{1}^{\prime}$. But the quasiequation (e7), interpreted in the obvious way, says that $x_{0}^{\prime}=x_{1}^{\prime}$, a contradiction.

So, the sequence $F_{1}, p_{1}, \ldots$ ends with $F_{r}, p_{r}$ for some $r \leqslant K-1$. Clearly, every subset of the form $q^{-1}(F)$ for a basic polynomial $q$ can be found among $F_{1}, \ldots, F_{r}$. Put $O=B-\left(F_{1} \cup \ldots \cup F_{r}\right)$, so that $0 \in O$ and $F_{1}, \ldots, F_{r}, O$ is a partition of $B$. It remains to prove that the corresponding equivalence is a congruence of $B$.

Suppose that there exist an $n$-ary operation $f$ in $\sigma$ and an $n$-tuple $a_{1}, \ldots, a_{n}$ of elements of $B$ such that $a_{j} \in O$ for some $j$ but $f\left(a_{1}, \ldots, a_{n}\right) \in F_{i}$ for some $i$. Then $p\left(a_{j}\right) \in F_{i}$ where $p(x)=f\left(a_{1}, \ldots, a_{j-1}, x, a_{j+1}, \ldots, a_{n}\right)$ is a basic polynomial of depth 1 and $a_{j} \notin F_{1} \cup \ldots \cup F_{r}$, so that $\left(q_{i} p\right)^{-1}(F)$ is nonempty and different from all $F_{1}, \ldots, F_{r}$, a contradiction. We have proved that if at least one of the elements $a_{1}, \ldots, a_{n}$ belongs to $O$, then $f\left(a_{1}, \ldots, a_{n}\right) \in O$.

Now it remains to show that if $f$ is $n$-ary, $f\left(a_{1}, \ldots, a_{n}\right) \in F_{j}$ and $a_{i}, a_{i}^{\prime} \in F_{k}$ for some $j, k \in\{1, \ldots, r\}$ and $i \in\{1, \ldots, n\}$, then $f\left(a_{1}, \ldots, a_{i-1}, a_{i}^{\prime}, a_{i+1}, \ldots, a_{n}\right) \in F_{j}$. Put $q(x)=q_{j}\left(f\left(a_{1}, \ldots, a_{i-1}, x, a_{i+1}, \ldots, a_{n}\right)\right)$, so that $q$ is a basic polynomial of $B$ of depth at most $K$. We have $q\left(a_{i}\right) \in F$ and $q_{k}\left(a_{i}\right) \in F$, so that $q^{-1}(F)=q_{k}^{-1}(F)$. Since $a_{i}^{\prime}$ belongs to this set, we get $q\left(a_{i}^{\prime}\right) \in F$, i.e., $f\left(a_{1}, \ldots, a_{i-1}, a_{i}^{\prime}, a_{i+1}, \ldots, a_{n}\right) \in F_{j}$. 
Theorem 3.4. Let $A$ be a finite compatible, flat algebra with $K$ elements. Then $Q_{A}^{\prime}$ is a finitely $q$-based and locally finite quasivariety containing $A$; every algebra in $Q_{A}^{\prime}$ is isomorphic to a subdirect product of algebras of cardinality at most $K$. Consequently, $A$ is not inherently nonfinitely q-based.

P r o of. Let $B \in Q_{A}^{\prime}$. For every pair $b_{0}, b_{1}$ of distinct elements of $B$ (we can assume that $b_{1} \nless b_{0}$ ) there exists a maximal filter of $B$ containing $b_{1}$ but not $b_{0}$, so that by Lemma 3.3 these two elements can be separated by a congruence with at most $K$ blocks. It follows that every algebra from $B$ is isomorphic to a subdirect product of algebras of cardinality at most $K$. Thus $Q_{A}^{\prime}$ is contained in a finitely generated variety and hence it is locally finite. According to Lemma 3.1, $Q_{A}^{\prime}$ is finitely $q$-based and contains $A$.

\section{Finitely $q$-Based compatible flat algebras}

Let $A$ be a finite compatible flat algebra. By a segment of $A$ we will mean a nonempty subset of $A$, the elements of which can be arranged into a finite sequence $0, c_{1}, \ldots, c_{r}$ in such a way that $c_{1} \neq 0$ and for every $i=2, \ldots, r$ there exists a basic polynomial $p$ of $A$ of depth 1 with $p\left(c_{i}\right)=c_{j}$ for some $j \in\{1, \ldots, i-1\}$.

Let $S$ be a segment of $A$. The algebra obtained from $S$, considered as a partial subalgebra of $A$, by setting all the undefined operations to 0 will be called the 0-completion of $S$.

Let $S$ be a segment of $A$ and $S^{\prime}$ be the subalgebra of $A$ generated by $S$. The segment $S$ is said to be regular if the equivalence on $S^{\prime}$ with the only non-singleton block $\{0\} \cup\left(S^{\prime}-S\right)$ is a congruence of $S^{\prime}$. In that case, the factor of $S^{\prime}$ by this congruence is isomorphic to the 0-completion of $S$.

Theorem 4.1. Let $A$ be a finite compatible flat algebra such that the 0-completion of every regular segment of $A$ belongs to the quasivariety generated by $A$. Then $A$ is finitely $q$-based.

Proof. Denote by $Q_{A}^{\prime \prime}$ the subquasivariety of $Q_{A}^{\prime}$ determined by the quasiequations (1)-(7) and all quasiequations in at most $K$ variables that are satisfied in $A$. (Here $K=|A|$.) Since $Q_{A}^{\prime}$ is locally finite by Theorem 3.4, $Q_{A}^{\prime \prime}$ is locally finite. Since only finitely many equations are needed to reduce the terms in at most $K$ variables to a finite set $T_{0}$ of such terms, and then quasiequations in at most $K$ variables correspond to subsets of $T_{0}^{2}$ with distinguished elements, $Q_{A}^{\prime \prime}$ is finitely $q$-based. Of course, $A \in Q_{A}^{\prime \prime}$. We are going to prove that $Q_{A}^{\prime \prime}$ is the quasivariety generated by $A$. It is sufficient to show that every finite algebra from $Q_{A}^{\prime \prime}$ belongs to the quasivariety generated by $A$. 
Let $B$ be a finite algebra from $Q_{A}^{\prime \prime}$; let $b_{0}, b_{1} \in B$ be such that $b_{1} \nless b_{0}$. By 3.3 there is a congruence with at most $K$ blocks $O, F_{1}, \ldots, F_{r}$, yielding a quotient algebra $C$, such that $F_{1}, \ldots, F_{r}$ are filters (now they are principal filters), $F_{1}=F$, $b_{1} \in F_{1}, b_{0} \in O$, and for every $i \in\{2, \ldots, r\}$ there exist an index $j<i$ and a basic polynomial $p_{i}$ of length 1 with $F_{i}=p_{i}^{-1}\left(F_{j}\right)$. But all the coefficients occurring in $p_{i}$ belong to $F_{1} \cup \ldots \cup F_{r}$, so there exists a basic $x$-term $u_{i}\left(x, x_{1}, \ldots, x_{r}\right)$ of depth 1 such that $u_{i}\left(F_{i}, F_{1}, \ldots, F_{r}\right) \subseteq F_{j}$. Now we can combine these terms $u_{i}$ together to obtain, for each $i$, a basic $x$-term $t_{i}\left(x, x_{1}, \ldots, x_{r}\right)$ such that $t_{i}\left(F_{i}, F_{1}, \ldots, F_{r}\right) \subseteq F$, i.e., $t_{i}^{C}\left(F_{i}, F_{1}, \ldots, F_{r}\right)=F_{1}$. (We take $t_{1}=x$.) For any term $u$ denote by $t_{i}(u)$ the term obtained from $t_{i}$ by replacing the only occurrence of $x$ with $u$. Now consider the quasiequation

$$
x_{0} \leqslant x_{1} \& D \rightarrow x_{0}=x_{1}
$$

where $D$ is the conjunction of all these equations:

(i) $t_{i}\left(x_{i}\right) \geqslant x_{1}$, for any $i=1, \ldots, r$;

(ii) $t_{i}\left(x_{j}\right) \wedge x_{1} \leqslant x_{0}$, for any $i, j \in\{1, \ldots, r\}$ with $i \neq j$;

(iii) $t_{i}\left(f\left(x_{i_{1}}, \ldots, x_{i_{n}}\right)\right) \geqslant x_{1}$, for any $n$-ary operation $f$ of $\sigma$ and any $i, i_{1}, \ldots, i_{n}$ with $f^{C}\left(F_{i_{1}}, \ldots, F_{i_{n}}\right)=F_{i}$

(iv) $t_{i}(u) \wedge x_{1} \leqslant x_{0}$, for any $i=1, \ldots, r$ and any term $u$ in variables $x_{1}, \ldots, x_{r}$ containing a subterm $f\left(x_{i_{1}}, \ldots, x_{i_{n}}\right)$ with $f^{C}\left(F_{i_{1}}, \ldots, F_{i_{n}}\right)=O$ (it is possible to consider only finitely many such terms $u$ ).

Clearly, this quasiequation fails in $B$; since it is a quasiequation in at most $K$ variables $x_{0}, \ldots, x_{r}$, it must fail in $A$ by some elements $a_{0}, a_{1}, \ldots, a_{r}$. But then the subset $\left\{a_{0}, a_{1}, \ldots, a_{r}\right\}$ is a regular segment of $A$, and the 0 -completion of this subset is isomorphic to $C$. Since $C$ belongs to the quasivariety generated by $A$, the elements $b_{0}, b_{1}$ were separated by a congruence, the factor by which belongs to the quasivariety.

Corollary 4.2. Every finite flat digraph algebra is finitely q-based.

P roof. In this case, all segments are subalgebras.

Corollary 4.3. The flat algebra over any finite quasigroup (considered as a groupoid) is finitely q-based.

P r o of. In this case, all regular segments are subalgebras.

Corollary 4.4. If $\sigma$ is the signature containing only one unary symbol in addition to $\wedge$ and 0 , then every finite compatible flat $\sigma$-algebra is finitely $q$-based.

Proof. In this case, the 0-completion of every segment is isomorphic to a subalgebra. 


\section{The EMBedding Theorem}

Theorem 5.1. Let $\sigma$ be a finite signature containing, in addition to $\wedge$ and 0 , at least two unary symbols $f$ and $g$ (and, possibly, some other operation symbols). Then every finite compatible flat $\sigma$-algebra can be embedded into two finite compatible flat $\sigma$-algebras, one finitely $q$-based and the other one not finitely $q$-based.

P r o of. Let $G$ be a finite compatible flat algebra.

Denote by $S_{1}, \ldots, S_{r}$ all the segments of $G$. (It would be sufficient to take just those with the 0 -completions not belonging to the quasivariety generated by $G$.) For every $i=1, \ldots, r$ let us take an isomorphic copy $T_{i}$ of the partial algebra $S_{i}-\{0\}$, in such a way that the sets $G, T_{1}, \ldots, T_{r}$ are pairwise disjoint. Denote by $G^{\prime}$ the flat algebra with the underlying set $G \cup T_{1} \cup \ldots \cup T_{r}$, with the operations evaluated to 0 in all cases except when needed to define them in such a way that $G$ is a subalgebra and $T_{i}$ are partial subalgebras. It follows from Theorem 4.1 that $G^{\prime}$ is finitely $q$-based.

Next we are going to construct a non-finitely $q$-based extension of $G$. Let us take one fixed positive integer $k$ such that $k \geqslant 2$ and there is no sequence $u_{0}, u_{1}, \ldots, u_{k}$ of pairwise distinct elements of $G-\{0\}$ such that $g\left(u_{i-1}\right)=u_{i}$ for $i=1, \ldots, k$. Denote by $A$ the flat algebra, with $G$ as a subalgebra, containing $k+10$ additional elements $u_{0}, u_{1}, \ldots, u_{k}, a, b, c, v_{2}, a_{2}, b_{2}, v_{3}, a_{3}, c_{3}$ with all operations not inside $G$ evaluated to 0 except for

$$
\begin{gathered}
g\left(u_{i-1}\right)=u_{i} \quad \text { for } i=1, \ldots, k \\
f\left(u_{0}\right)=a, \quad f(a)=b, \quad g(a)=c, \\
f\left(v_{2}\right)=a_{2}, \quad f\left(a_{2}\right)=b_{2}, \quad f\left(v_{3}\right)=a_{3}, \quad g\left(a_{3}\right)=b_{3} .
\end{gathered}
$$

(Fig. 1, in which the elements not belonging to $G$ are pictured for $k=2$, may help to understand this definition.)
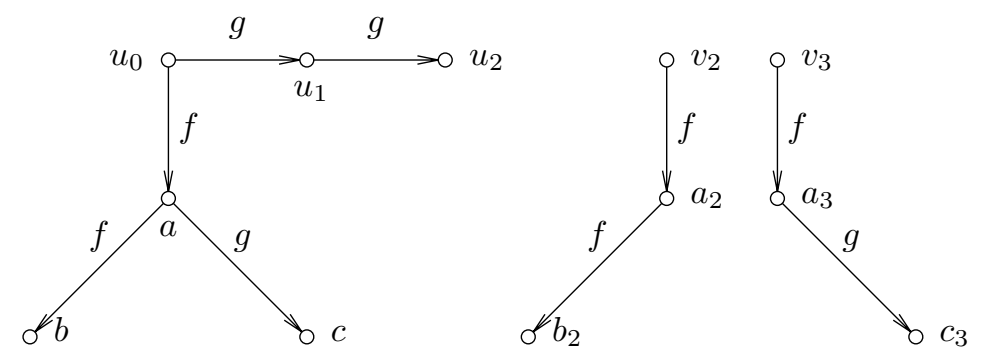

Fig. 1

Denote by $Q$ the quasivariety generated by $A$. A $\sigma$-algebra $B$ belongs to $Q$ if and only if every two distinct elements of $B$ can be separated by a homomorphism of $B$ into $A$. 
For every positive integer $n$ let $A_{n}$ be the $\sigma$-algebra with elements $0, u_{0}, \ldots, u_{k}$, $\alpha_{i, j}, \beta_{i}, \gamma_{j}(0 \leqslant i \leqslant n, 0 \leqslant j \leqslant n-1, i-1 \leqslant j \leqslant i)$ and with operations defined in this way: $A_{n}$ is a semilattice with the only comparabilities $0<u_{i}(i=$ $0, \ldots, k), 0<\beta_{n}<\beta_{n-1}<\ldots<\beta_{0}, 0<\gamma_{n-1}<\gamma_{n-2}<\ldots<\gamma_{0}, 0<\alpha_{n, n-1}<$ $\alpha_{n-1, n-1}<\alpha_{n-1, n-2}<\ldots<\alpha_{1,0}<\alpha_{0,0}$; the other operations evaluate to 0 except that $g\left(u_{i-1}\right)=u_{i}(i=1, \ldots, k), f\left(u_{0}\right)=\alpha_{0,0}, f\left(\alpha_{i, j}\right)=\beta_{i}, g\left(\alpha_{i, j}\right)=\gamma_{j}$. (Fig. 2, in which the situation is illustrated for $k=2$ and $n=3$, may help to understand this definition. In the picture lines with arrows indicate unary operations, while the other lines represent coverings but the covers between 0 and the elements $u_{i}$ are not indicated.)

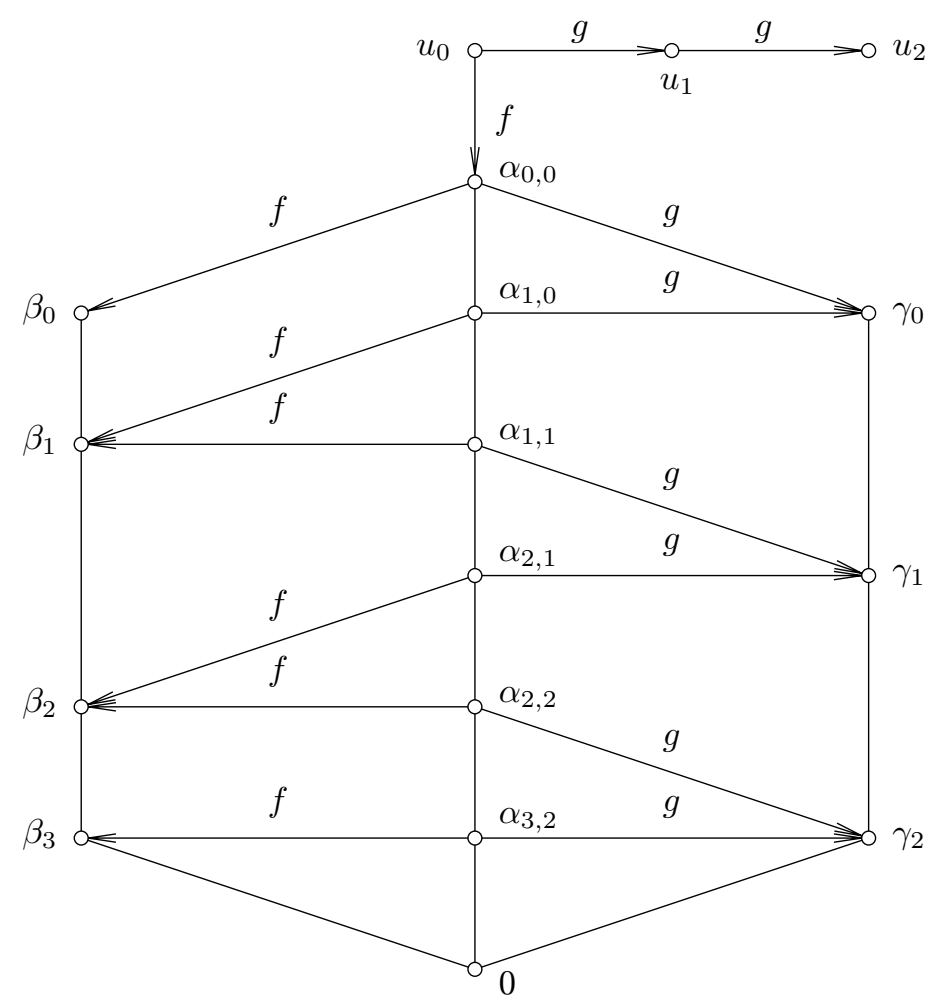

Fig. 2

Denote by $r_{n}$ the equivalence on $A_{n}$ with the only non-singleton block $\left\{0, \beta_{n}\right\}$. Clearly, $r_{n}$ is a congruence of $A_{n}$. Denote the factor $A_{n} / r_{n}$ by $B_{n}$. For $a \in A_{n}-$ $\left\{0, \beta_{n}\right\}$, the element $a / r_{n}$ will be identified with $a$.

Suppose that there exists a homomorphism $H: B_{n} \rightarrow A$ such that $H\left(u_{k}\right) \neq$ $H\left(0 / r_{n}\right)$, i.e., $H\left(u_{k}\right) \neq 0$. Since $g^{k}\left(u_{0}\right)=u_{k}$ in $B_{n}$ and there is no other element $e$ 
in $A$ with $g^{k}(e) \neq 0$ and $g^{k+1}(e)=0$ other than $u_{0}$, we get $H\left(u_{0}\right)=u_{0}$ and then $H\left(u_{i}\right)=H\left(g^{i}\left(u_{0}\right)\right)=g^{i}\left(H\left(u_{0}\right)\right)=g^{i}\left(u_{0}\right)=u_{i}$ for all $i$. Now $H\left(\alpha_{0,0}\right)=H\left(f\left(u_{0}\right)\right)=$ $f\left(H\left(u_{0}\right)\right)=f\left(u_{0}\right)=a$. Consequently, $H\left(\beta_{0}\right)=b$ and $H\left(\gamma_{0}\right)=c$. Since $g\left(\alpha_{1,0}\right)=\gamma_{0}$ and $a$ is the only element of $A$ with $g(a)=c$, it follows that $H\left(\alpha_{1,0}\right)=a$. If $H\left(\alpha_{i, i-1}\right)=a$ for some $i<n$, then using $f$ in a similar way we can show that $H\left(\alpha_{i, i}\right)=a$, and then using $g$ to show that $H\left(\alpha_{i+1, i}\right)=a$. By induction we get $H\left(\alpha_{n, n-1}\right)=a$. But then $H\left(0 / r_{n}\right)=H\left(\beta_{n} / r_{n}\right)=H\left(f\left(\alpha_{n, n-1}\right)\right)=f(a)=b$, a contradiction.

Since the element $u_{k}$ cannot be separated from $0 / r_{n}$ by a homomorphism of $B_{n}$ into $A$, we conclude that $B_{n}$ does not belong to $Q$.

Let $\alpha_{m, m^{\prime}}$ be an element of $B_{n}$ such that $0<m<n$. Clearly, the set $C=$ $B_{n}-\left\{\alpha_{m, m^{\prime}}\right\}$ is a subalgebra of $B_{n}$. We are going to prove that $C$ belongs to $Q$. For this purpose, it is sufficient to show that whenever $e, e^{\prime}$ are two elements of $C$ such that $e$ is covered by $e^{\prime}$, then $e, e^{\prime}$ can be separated by a homomorphism of $C$ into $A$.

For every $i \leqslant n-1$ define a mapping $\psi_{i}$ of $B_{n}$ into $A$ by $\psi_{i}\left(u_{0}\right)=v_{2}, \psi_{i}(e)=a_{2}$ for $e \geqslant \alpha_{i, i}, \psi_{i}(e)=b_{2}$ for $e \geqslant \beta_{i}$ and $\psi_{i}(e)=0$ for all other elements $e$. Also, for every $i \leqslant n-1$ define a mapping $\chi_{i}$ of $B_{n}$ into $A$ by $\chi_{i}\left(u_{0}\right)=v_{3}, \chi_{i}(e)=a_{3}$ for $e \geqslant \alpha_{i+1, i}, \chi_{i}(e)=c_{3}$ for $e \geqslant \gamma_{i}$ and $\chi_{i}(e)=0$ for all other elements $e$. It is easy to check that both $\psi_{i}$ and $\chi_{i}$ are homomorphisms. Consequently, their restrictions to $C$ are homomorphisms of $C$ into $A$. The only pairs of covers not separated by any of these homomorphisms are the pairs $\left(0, u_{1}\right), \ldots,\left(0, u_{k}\right)$. So, it remains to separate these pairs of elements.

If $m=m^{\prime}$, then these pairs are separated by the homomorphism $\varphi$ defined in this way: $\varphi\left(u_{0}\right)=u_{0}, \ldots, \varphi\left(u_{k}\right)=u_{k}, \varphi(e)=a$ for $e \geqslant \alpha_{m, m-1}, \varphi(e)=b$ for $e \geqslant \beta_{m}$, $\varphi(e)=c$ for $e \geqslant \gamma_{m-1}$ and $\varphi(e)=0$ for all other elements $e$. If $m^{\prime}=m-1$, then they are separated by the homomorphism $\varphi^{\prime}$ defined in this way: $\varphi^{\prime}\left(u_{0}\right)=$ $u_{0}, \ldots, \varphi^{\prime}\left(u_{k}\right)=u_{k}, \varphi^{\prime}(e)=a$ for $e \geqslant \alpha_{m^{\prime}, m^{\prime}}, \varphi^{\prime}(e)=b$ for $e \geqslant \beta_{m^{\prime}}, \varphi^{\prime}(e)=c$ for $e \geqslant \gamma_{m^{\prime}}$ and $\varphi^{\prime}(e)=0$ for all other elements $e$.

We have proved that $C$ belongs to $Q$. Since every subalgebra of $B_{n}$ generated by at most $n-k$ elements is contained in at least one such $C$, it follows that every subalgebra generated by at most $n-k$ elements belongs to $Q$. Consequently, there is no base for the quasiequations of $Q$ that would contain only quasiequations in at most $n-k$ variables. Since $k$ was fixed while $n$ was arbitrary, there is no finite base at all.

Remark 5.2. In the above construction of the algebra $A$ it was not essential that the elements $b_{2}$ and $c_{3}$ are distinct. 


\section{References}

[1] J. Lawrence and R. Willard: On finitely based groups and nonfinitely based quasivarieties. J. Algebra 203 (1998), 1-11.

[2] R. McKenzie: The residual bounds of finite algebras. Internat. J. Alg. Comput. 6 (1996), $1-28$.

[3] R. McKenzie: The residual bound of a finite algebra is not computable. Internat. J. Alg. Comput. 6 (1996), 29-48.

[4] R. McKenzie: Tarski's finite basis problem is undecidable. Internat. J. Alg. Comput. 6 (1996), 49-104.

[5] R. McKenzie, G. McNulty and W. Taylor: Algebras, Lattices, Varieties, Vol. I. Wadsworth \& Brooks/Cole, Monterey, 1987.

[6] D. Pigozzi: Finite basis theorems for relatively congruence-distributive quasivarieties. Transactions of the AMS 310 (1988), 499-533.

Authors' addresses: J. J e ž e k, MFF UK, Sokolovská 83, 18600 Praha 8, Czech Republic, e-mail: jezek@karlin.mff.cuni.cz; M. Maróti and R. M c Kenzie, Department of Mathematics, Vanderbilt University, 1326 Stevenson Center, Nashville, TN 37240, e-mails: miklos.maroti@vanderbilt.edu, ralph.n.mckenzie@vanderbilt.edu. 